\title{
AGE SUSCEPTIBIIITY TO FAMILIAL INFECTION IN RHEUMATIC FEVER
}

\author{
BY JOHN R. PAUL
}

\begin{abstract}
(From the Department of Internal Medicine, Yale University and the New Haven Dispensary, New Haven)
\end{abstract}

(Received for publication November 11, 1930)

One expression of our changing views with regard to rheumatic fever is the increasing importance being attached to juvenile forms of the disease. Older figures on the age incidence of this disease were based largely upon the incidence of both the primary, and recurrent attacks. Such figures minimized the importance of childhood as the period of greatest susceptibility. For instance in Rolly's monograph on rheumatic fever (1), published in 1920, he listed the age incidence of all periods of activity in a series of 1450 cases, and from these figures found, that only 4 per cent of the attacks occurred in the first decade of life; 40 per cent in the second; 39 per cent in the third; etc. The conclusion reached was that rheumatic fever found its highest incidence in the second and third decades of life. If, on the other hand, a series of cases is analyzed for the age incidence of first attacks, a very different result is obtained, showing that by far the greater number of initial manifestations of the disease occur in the first decade of life. This fact now seems well established among groups of patients attending hospital clinics in large cities, although we have no data on rural populations.

Our present views with regard to the age incidence of first attacks may now be briefly reviewed as follows: it is apparently rare, although not exceptional, to find the disease during the first year of life. Most of the cases which have been recorded in young infants were being nursed by mothers who were suffering from active phases of the disease, a fact which is of importance not only from the standpoint of age susceptibility but also from that of the transmissibility of the disease. Equally important are the isolated case reports, mentioned by Richdorf and Griffith (2), of apparent congenital acquisition of rheumatic 
fever. These describe instances of the disease occurring in women who were pregnant, to be followed by the birth of an infant who within one or a few days apparently showed manifestations of rheumatic fever.

After the age of one year there is a rapid rise in the incidence of first attacks, reaching a peak between 5 and 15 years. In England, Poynton's series (3) showed an average age at onset of 7; Coombs' series (4) of 10. In New York City, Wilson et al. (5), who made extensive

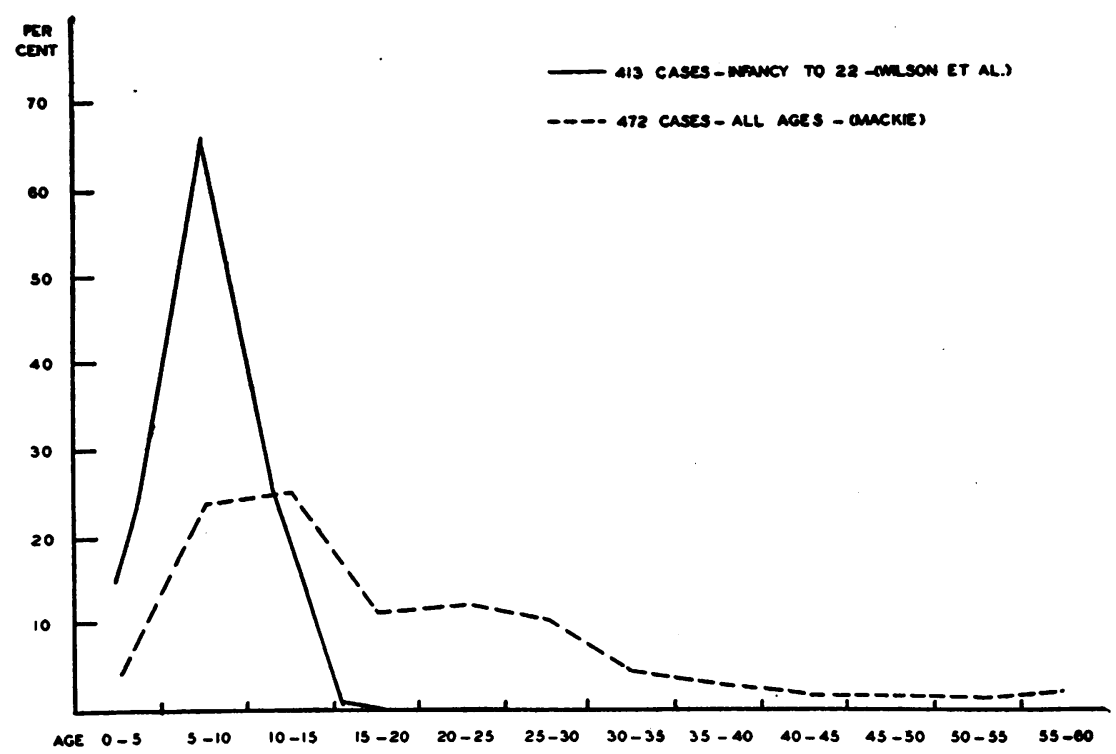

Fig. 1. The Age Incidence of First Attacks of Rheumatic Fever

statistical studies on a series of 413 children, ranging in age from infancy up to 22 years, observed during a ten-year period, that in 98.4 per cent of their cases, initial infection occurred before the age of 15; the average age at onset was 7.3 years. Mackie (6), also in New York City, has attempted to determine the age incidence of initial attacks from a series of $\mathbf{4 7 2}$ individuals, drawing on historical data for his facts. The results of both of these analyses are shown in figure 1. Their differences may be dependent both upon the manner in which the data were obtained, and the manner in which the series of cases 
were selected, but both emphasize the 5-15-year period as one in which the disease is generally acquired. The attempt has also been made to decide from these and other data, just where susceptibility to rheumatic fever reaches its height and where it begins to diminish. Wilson et al. (5) believe that rheumatic infection, concerns itself primarily with children of the grade school age of 6 to 14, and that about or before the age of twelve, the tendency to infection begins to diminish.

Caution must be exercised in making generalizations from the figures quoted above when we recall for instance that Wilson's group was drawn from a juvenile population in which the factor of initial selection is of the utmost importance; for if rheumatic fever adheres to the principles guiding the spread of other infectious diseases, it owes its incidence to many factors, including the facility with which it may be spread, as well as the size and character of the susceptible population.

With these points in view it has seemed interesting to analyze this situation as it occurs in families in which the incidence of the disease is high. Here again one is dealing with a rigidly selected population but the data would seem to be valuable because, besides determining the age incidence of initial attacks in the family series, one has the further opportunity of listing the ages at which exposure to familial infection, first and subsequently occurred.

\section{METHODS}

The method of selecting the families and of assembling and recording the data, has been described in the preceding paper (7). Fourteen families were available for the present study, representing 99 individuals: 24 parents and 75 children, covering an age group extending from infancy up to 50 years, although a gap occurs between the ages of 18 and 33. In order to facilitate our statistical analysis, a chart was devised on which each of the individuals has been designated by a vertical line, corresponding in length with the individual's age. These lines have been punctuated by legends, designating the following three episodes: (a) Exposure to familial infection. This has been defined as follows: when any member of a given household suffered from an active phase of rheumatic fever it was presumed that all of the 
other members living at home at that time, were exposed. As some of these active phases were prolonged, it was often difficult to say just when exposure ended and whether multiple exposures occurred. Such prolonged attacks have, however, been recorded as a single

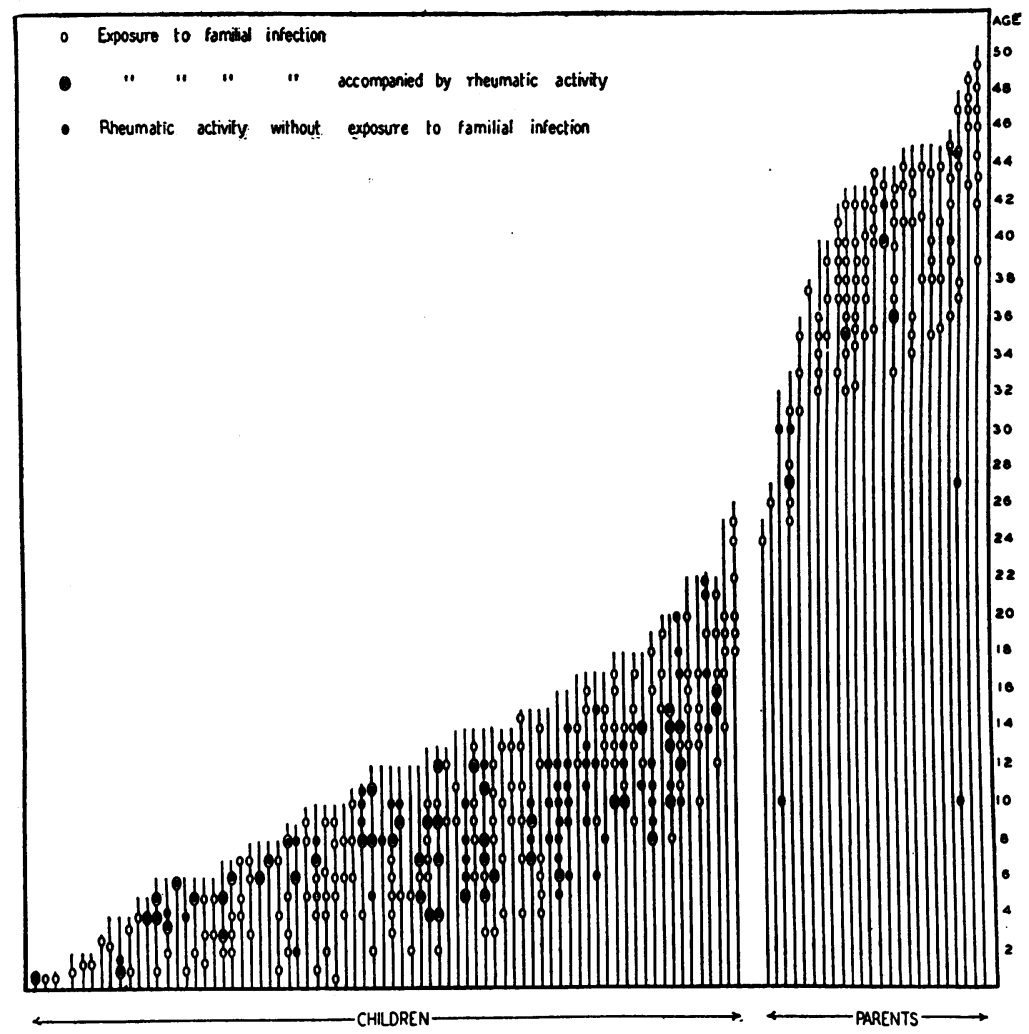

Fig. 2. The Age Distribution of Attacks of Rheumatic Fever in 99 Individuals, with Special ReFerence to AcQuisttion of the Disease Following Fammial Exposure

exposure dated from the onset of the attack. (b) Exposure to familial infection as defined above, with acquisition of the disease. Instances of this kind are represented by the development of an active phase of rheumatic fever in any member of the household within a period of 4 weeks following the development of the disease in another member of 
the family. In a few instances several cases have appeared almost simultaneously in a given family. All of these cases may have been examples of acquisition of the disease from extra-familial sources. Under these circumstances, I have assumed as an arbitrary principle, that one of the cases represented extra-familial infection, the others, intra-familial infection. (c) Acquisition of the disease, not explainable on the basis of familial infection. This has been defined as the appear-

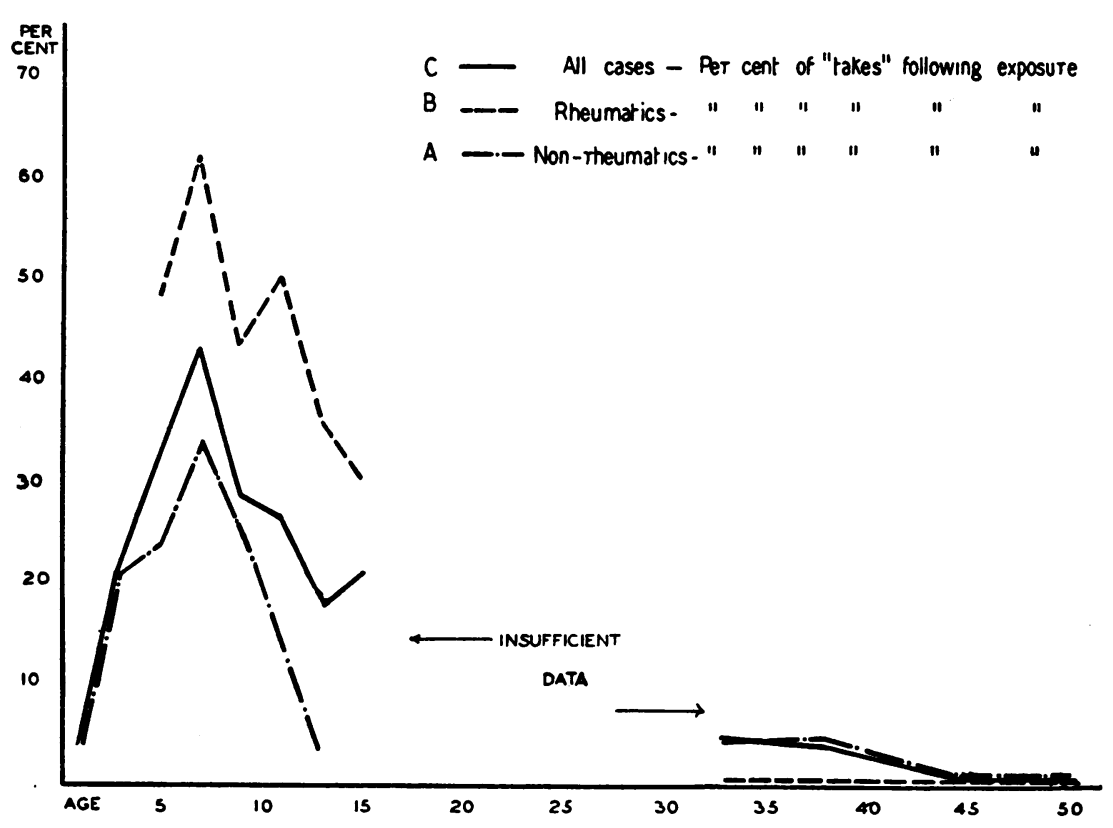

Fig. 3. The Percentage Rates at Which Individuals of Different Ages Acquired Rheumatic Fever, Following Familial Exposure

ance of a primary or recurrent active phase of the disease in a member of a household, in which no other members of the family group had shown active evidences of rheumatic fever within a period of 6 weeks.

\section{RESULTS}

In figure 2 is shown a chart constructed on the basis just described. It shows the age distribution of the cases, and roughly the period during which they have been under observation. It shows a concen- 
tration of active phases of the disease during the ages of 4-15 years and its relative scarcity in the parental group, in spite of repeated familial exposure.

On the basis of data in this chart curves have been drawn representing the degree of susceptibility following exposure to familial infection. These are shown in figure 3. The gap which exists between the ages of 18 and 33, is due to the manner in which cases were selected. The curves in figure 3 show the percentage rates at which three groups of individuals developed active phases of the disease following exposure to familial infection; in (A), individuals who had not previously suffered from any manifestation of rheumatic fever; (B), individuals who had previously suffered from some manifestation of rheumatic fever; (C) all individuals. The first curve (A) conforms with Wilson's estimation of the age incidence of initial attacks (cf. fig. 1). Curve (B) shows that susceptibility to recurrence is at least twice that of the susceptibility to first attacks, and this susceptibility continues into the second decade. In the parents it will be noted that in all groups, acquisition of the disease following familial exposure, occurred in less than 5 per cent, between the ages of 33-45, and an even lower figure is recorded between $45-50$.

\section{DISCUSSION}

Caution has already been expressed with regard to making generalizations from data of this type. We are here concerned with a group of Dispensary patients heavily exposed to the disease, living under poor hygienic surroundings, and under conditions which seem to predispose to the spread of the disease. A different age distribution might well occur in other populations, particularly if one were to include groups of individuals from rural districts, or recent immigrants, from regions where the disease is rare, who probably had not been heavily exposed during childhood. This point has been discussed by Andrieu (8); and by Hathaway (9), who had the opportunity of studying an epidemic of rheumatic fever among students at the University of Minnesota, in which 60 per cent of the cases occurred among a relatively small segregated group of Agricultural students composing less than 10 per cent of the student population. Hathaway does not mention whether any of this group had previously suffered from 
manifestations of the disease, but calls attention to the fact that most of the agricultural students came from rural areas and probably had had fewer previous contacts. Similar observations of this same general type have been made by Dr. Boas of New York City, who has noted that rheumatic fever, developing in Porto Rican adults, who were recent immigrants to the city, may often assume the severe fulminating form, which in urban populations we are accustomed to see in childhood (10). The author has also seen instances of this type, in adult negroes who had recently emigrated from the south to a large northern city.

Notwithstanding these considerations, however, the fact remains that in the children in our series of rheumatic families, the spread of the disease occurred far more frequently in the age group of 4-12 years. Susceptibility in exposed children detected by this method reaches a sharp peak at the age of 7 years which is an even earlier age than that determined by Wilson and her collaborators.

The conclusions to which one is drawn are, that under the observed social and living conditions, and with this method of selection, children between the age of 5-12, living in a family in which another member was suffering from an active phase of the disease, had a 25-35 per cent chance of acquiring the disease. In fact their chances of acquiring the disease were considerably higher than that of acquiring rabies had they been bitten by a rabid dog and left untreated. If they had already suffered from an initial attack the chances of suffering from a recurrence in this environment were almost doubled, and this enhanced susceptibility continued through adolescence.

Strong preventive therapeutic indications would seem to exist in these observations, calling for the protection of children under 12 years of age, from this type of environment.

\section{SUMMARY}

In a selected population representing members of families, in which two or more individuals had suffered from evidences of rheumatic fever, it has been found that the disease spread particularly to the younger members of the family, and was largely distributed throughout the age group 5-12, reaching its peak at about the age of 7 .

Among the children between 5 and 12, who apparently had not 
previously suffered from any manifestations of the disease, rheumatic fever was acquired in association with familial exposure, at the rate of 25-35 per cent.

Among children between 5 and 12 years of age who had already survived an initial attack, recurrences were noted in association with familial exposure at the rate of 35-60 per cent, decreasing as age increased, but representing a markedly enhanced susceptibility during adolescence.

\section{BIBLIOGRAPHY}

1. Rolly, F., Berlin, 1920. Der akute Gelenkrheumatismus.

2. Richdorf, L. F., and Griffith, W. H., Am. J. Dis. Child., 1926, xxxi, 250. Polyarthritis. Report of a Case in an Infant Six Days Old Associated with Acute Rheumatic Fever in the Mother.

3. Poynton, F. J., Brit. Med. J., 1918, i, 249. Observations on the Nature and Symptoms of Cardiac Infection in Childhood.

4. Coombs, Carey F., Lancet, 1927, i, 579 and 634. Rheumatic Infection in Childhood.

5. Wilson, M. G., Lingg, C., and Croxford, G., Am. Heart J., 1928, iv, 164. Statistical Studies Bearing on Problems in the Classification of Heart Diseases. III. Heart Disease in Children.

6. Mackie, T. T., Am. J. Med. Sci., 1926, clxxii, 199. An Analytical Study on 393 Cases of Rheumatic Fever and 89 Cases of Chorea.

7. Paul, J. R. and Salinger, R., J. Clin. Invest., 1931, x, 33. The Spread of Rheumatic Fever through Families.

8. Andrieu, G., Toulouse, 1926. Le Rhumatisme Articulaire Aigu. Maladie contagieuse.

9. Hathaway, J. C., Minnesota Med., 1930, xiii, 37. Rheumatic Fever. A Communicable Disease.

10. Boas, E. P., Personal Communication. 